\title{
Tunnel use facilitates handling of ICR mice and decreases experimental variation
}

\author{
Yu NAKAMURA ${ }^{1)}$ and Kaoru SUZUKI ${ }^{1) *}$ \\ 1)Field Science Center, Tokyo University of Agriculture and Technology, 3-5-8 Saiwai-cho, Fuchu, \\ Tokyo 183-8509, Japan
}

J. Vet. Med. Sci.

80(6): 886-892, 2018

doi: 10.1292/jvms.18-0044

Received: 25 January 2018

Accepted: 3 April 2018

Published online in J-STAGE:

13 April 2018
ABSTRACT. We evaluated a handling method using tunnels to tame laboratory mice (ICR) in the context of animal welfare and ease of handling. During 1-week acclimation to handling and subsequent 1-week oral administration (once per day), voluntary interaction with the experimenter was much greater in mice handled by a tunnel compared to those picked up by the tail. According to a rating of the ease of handling laboratory mice, a tunnel facilitated mouse handling during acclimation to handling and oral gavage of saline compared to tail handling. In addition, mice handled by a tunnel showed less anxiety than mice handled by the tail in the open field test, but not in elevated plus maze. Calculation of experimental variation in behavioral tests, which were used to mimic pharmacological studies, suggested that mice handled by a tunnel exhibited the tendency of less variation compared to those picked up by the tail, in both groups that were intraperitoneally administered saline as placebo and diazepam as an active drug. Thus, tunnel use could be beneficial for improving animal welfare and facilitated handling of ICR mice in mouse studies.

KEY WORDS: animal welfare, data variation, ease of handling, mice, tunnel handling

Routine procedures such as handling, restraint, and injections are known to cause an acute stress response in experimental animals [10]. Handling procedures could confound experimental results due to the uncertain effect of handling stress. Therefore, animals are tamed to the experimenters and experimental procedures by handling before experiments in order to control for confounding effects on results and improve animal welfare [9]. Tameness is defined as increased interaction of an animal with humans, and is a behavioral characteristic with two potential components: "reluctance to avoid humans" and "motivation to approach humans", and it is also associated with exploratory behavior [5, 13]. Hurst and West [7] clarified that mice handled by a tunnel (tunnel handling) preferred a handler on the ground of more increased voluntary interaction of mice with human, compared to mice picked up by the tail (standard tail handling). Voluntary interaction with human is similar to "motivation to approach human" in definition of Price [13]. In addition, tunnel handling showed lower anxiety in order to measure animal welfare, and decreased urination and defecation at handling, which were measured as an indicator of stress [7]. From these result, Hurst and West [7] recommended tunnel handing to reduce animal stress. In pharmacological studies, outbred ICR mice of 4-6 weeks of age are frequently subjected to repeated oral administration during handling [12, 18]. These procedures are known to cause rodents stress $[2,3]$. Therefore, it is necessary to examine whether tunnel handling can improve animal welfare during persistent stress such as during repeated oral drug administration.

Animal welfare procedures should be applied in ways that cause minimal interference with husbandry and care procedures $[1,8,16]$. A high degree of difficulty in animal handling due to more wildness of mice could be considered an added effort to experimenters. Although some studies have examined how new animal welfare procedures impact ease of handling of laboratory animals compared to standard procedures $[9,11]$, no studies have compared tunnel handling to standard tail handling in terms of difficulty of handling. Moreover, because of repeated oral administration, the degree of difficulty of handling would more increase remarkably in mice subjected to tunnel handling than in mice subjected to tail handling that mice are forced to capture by experimenters because mice subjected to tunnel handling would lose the willingness to enter a tunnel. It is important that the use of tunnel handling for animal welfare does not negatively impact ease of handling, as this would eliminate a possible reason for not implementing tunnel handling in animal studies. Wahlsten et al. [17] made rating scales for wildness of mice. These scales provide a good impression of the difficulties encountered while an experimenter would capture and hold laboratory mice that would possibly attempt to resist and escape. Thus, less wildness indicates ease of handling of laboratory mice.

Fridgeirsdottir [4] found that handled inbred C57BL/6 mice showed less data variation in behavioral tests than non-handled mice, suggesting that fewer mice would be needed to identify statistically significant differences. Gentle tunnel handling of mice

*Correspondence to: Suzuki, K.: kaoru@cc.tuat.ac.jp

O2018 The Japanese Society of Veterinary Science

This is an open-access article distributed under the terms of the Creative Commons Attribution Non-Commercial No Derivatives (by-nc-nd) License. (CC-BY-NC-ND 4.0: https://creativecommons.org/licenses/by-nc-nd/4.0/) 
might decrease data variation. In pharmacological studies, outbred ICR mice are frequently administered placebo for the control group or active drug for the experimental group. The administration of an active drug could enhance data variation by influencing the physiology and behavior of animals compared to placebo. Thus, it is necessary to examine whether tunnel handling can decrease data variation in mice administrated placebo and in those administered active drug, thereby facilitating the use of tunnel handling in pharmacological tests.

In this study, voluntary interaction of mice to the experimenter and ease of handling were compared between the tunnel handling group and the tail handling group in "experiment 1 ". While these were being evaluated, mice were handled daily for 1 week and administrated saline orally for a subsequent 1 week. In "experiment 2", anxiety-like behavior was assessed between handling groups using the open field and elevated plus maze tests. In addition, to mimic pharmacological studies in ICR mice, saline (placebo) and diazepam (active drug) were administrated to animals before the two behavioral tests, and the ability of tunnel handling to decrease behavioral variation in mice during experimental drug injection was investigated.

\section{MATERIALS AND METHODS}

\section{Animals and housing}

Eighty Jcl:ICR mice (20 days old) were obtained from CLEA Japan (Tokyo, Japan). Half of the mice were male, half were female. During the study, four mice were housed per cage under conventional conditions. All mice were maintained in identical plastic cages measuring W282 $\times$ H157 $\times$ D451 mm with woodchip bedding and a wire-top lid (CLEA Japan). Mice were provided with food (MF, Oriental Yeast Co., Tokyo, Japan) and water ad libitum. Cages were maintained at a temperature of $24 \pm 2{ }^{\circ} \mathrm{C}$ and $25-70 \%$ relative humidity and with a $12-\mathrm{hr}$ light/dark cycle, lights on at 6:00.

\section{Experimental design}

After mice were ear-punctured for individual identification at 20 days of age, they were separated by sex and randomly assigned to either the tunnel handling group (mice handled by a tunnel) or the tail handling group (mice handled by picking up the tail). These four groups were replicated five times with four mice per cage. For tunnel handling, the tunnel was brought towards the animal and held resting on the cage substrate while the animal was guided towards it with the other hand. Hands were cupped loosely over the tunnel ends to prevent escape until the mice were habituated to handling. Tunnels were transparent acrylic hollow cylinders, measuring $50 \mathrm{~mm}$ wide and $150 \mathrm{~mm}$ long (Sakura Plastics Co., Ltd., Yokohama, Japan). Tail handling consisted of grasping the tail base between the thumb and forefingers, and gently lifting the animal onto the experimenter's gloved hand or forearm. After 1 week handling, experiments were initiated when mice were 4 weeks old. Cages were cleaned on the last day of every week. "Experiment 1" included assessment of voluntary interaction with an experimenter and evaluation of ease of handling when mice were handled daily through 4 weeks of age and received gavage administration of saline to mimic a pharmacological procedure through 5 weeks of age. In "experiment 2", the open field and elevated plus maze tests were performed just after mice were intraperitoneally administrated saline as placebo or diazepam as active drug, when mice were 6 weeks of age. In addition, variation in behavioral test items was calculated for each group. The same experimenter performed all procedures in this study. All procedures were performed by trained personnel experienced in conducting animal studies. Permission for this study was obtained from the Tokyo University of Agriculture and Technology Laboratory Animal Care and Use Committee (approval number, 28-49).

\section{"Experiment 1"}

Handling sessions in mice through 4-5 weeks of age were carried out at 10:00-12:00.

\section{Handling of animals}

Handling sessions began when mice were 4 weeks of age. Mice were handled throughout seven sessions (one session per day). Each mouse was then held in the tunnel (tunnel handling group) or on the experimenter's hand (tail handling group) for 30 sec. After handling all mice in the cage, the experimenter moved away from the cage for $60 \mathrm{sec}$ before handling the mice a second time. In addition, each mouse was held for another $30 \mathrm{sec}$ so that each mouse was held for a total of $60 \mathrm{sec}$ per session. The experimenter wore a laboratory coat that was contaminated with mouse scent from previous handling sessions and gloves that were rubbed in soiled bedding from animals of the same sex. Tunnels were wiped clean with paper towel if contaminated by the animals during handling. The order that cages were handled was balanced across handling sessions. The experimenter counted the number of urination and defecation until experimenter released mice to a cage from starting to capture mice.

\section{Oral administration during handling}

When mice were 5 weeks of age, handling sessions included oral administration of saline (Otsuka Pharmaceutical Co., Ltd., Tokyo, Japan). Mice were administered saline by gavage once daily throughout seven sessions. After mice were captured by a tunnel or the tail and placed on the top of the cage, the animal was grasped by the back and picked up. Saline (5 ml/kg) was administered orally by an animal feeding needle (stainless steel, $1.2 \times 50 \mathrm{~mm}, 18 \mathrm{G}-50$; Natsume Seisakusho Co., Ltd., Tokyo, Japan). The experimenter counted the number of urination and defecation until experimenter released mice to a cage from starting to capture mice. 


\section{Interaction tests}

To measure the motivation of mice to approach the experimenter in anticipation of handling, voluntary interaction with the experimenter was assessed $60 \mathrm{sec}$ immediately before and after the 1, 4, 7, 8, 11 and 14 handling sessions (according to Hurst and West [7] with modifications). First, the handler motionlessly faced the cage for $60 \mathrm{sec}$. A gloved hand (tail handling group) or gloved hand holding a tunnel (tunnel handling group) was held resting in the cage without moving for 60 sec. Following interaction testing, the animals were acclimated to the handler at 4 weeks old and administered saline at 5 weeks old according to their respective handling method, after which the experimenter faced the cage again and performed a second interaction test for 60 sec. The duration of the following behaviors was recorded as voluntary interaction with the experimenter: sniffing (nose within $0.5 \mathrm{~cm}$ of gloved hand or a tunnel), inside tunnel (all four paws inside), climbing (all four paws on gloved hand or a tunnel), paw contact (front paws on gloved hand or a tunnel), peeking in tunnel (animal places front paws in tunnel and immediately retreats from tunnel). These behaviors were analyzed from video recordings (Canon IXY 210, Canon Inc., Tokyo, Japan) and stopwatch.

\section{Rating scale for ease of handling}

Ease of handling during handling sessions was measured using a rating scale for wildness that indicate the difficulty in capturing and holding mice, as devised by Wahlsten et al. [17]. Within each scale, ratings were ranked in order of the severity of the animal's reaction. Evaluations of capture and hold were performed twice at 4 weeks of age and once at 5 weeks of age per handling session. At 4 weeks of age, we utilized the higher rating that indicates a stronger resistance of mice between the first and second evaluations in a handling session. The high value in both scales showed low ease of handling.

\section{"Experiment 2"}

"Experiment 2" consisted of two behavioral tests and calculation of data variation of these tests. Half of the mice in each of the four different groups were administrated diazepam $(1.5 \mathrm{mg} / \mathrm{kg}$, Taiyo Pharmaceutical Industry, Nagoya, Japan) intraperitoneally in order to assess data variation. In addition, the other half of the mice were administrated saline (Otsuka Pharmaceutical Co., Ltd.) intraperitoneally as placebo, in order to examine whether we could detect the effects of diazepam on reducing anxiety-like behavior in both the tail and tunnel handling groups. Two of mice that were given diazepam have been fixed by ear-punch before a start of each experiment. Mice were administrated diazepam or saline intraperitoneally $30 \mathrm{~min}$ before each test. The interval between behavioral tests was $72 \mathrm{hr}$.

\section{Behavioral tests}

The open field and elevated plus maze tests were chosen as the behavioral tests. These tests have been routinely used for pharmacological studies of anti-anxiety agents.

\section{Open field test}

Mice were tested using a single 10-min trial during the light period between 10:00 and 15:00 at the beginning of 6 weeks of age. The open field was a square arena (W500 $\times$ H410 $\times$ D500 mm divided into 25 equal squares) on a white plastic floor and white plastic walls. The animals were placed in the center of the box, and the number of lines crossed with all four paws, and duration of time spent in the inner area (9 squares) were manually counted from video recordings (Canon IXY 210, Canon Inc.). At the end of testing, the mice were returned to their home cages according to their assigned handling method. The arena was cleaned with $70 \%$ ethyl alcohol after each test.

\section{Elevated plus maze test}

Mice were tested using a single 5-min trial during the light period between 10:00 and 15:00. The test arena consisted of a black plastic maze with two open and two closed arms (all $150 \mathrm{~mm}$ with side walls $150 \mathrm{~mm}$ high on the two closed arms) elevated 400 $\mathrm{mm}$ above the ground. The mice were brought to the center of the arena, facing an open arm for the 5-min test. The total time in open arms and the number of entries to open and all arms were scored. These behaviors were counted from video recordings (Canon IXY 210, Canon Inc.). At the end of testing, the mice were returned to their home cages according to their assigned handling method and the arena was wiped clean with $70 \%$ ethyl alcohol.

\section{Data analysis}

All values are expressed as means $\pm \mathrm{SD}$. The voluntary interaction of mice to approach the experimenter was determined by analysis of variance (ANOVA) with repeated measures using handling methods $\times$ session $\times$ before or after handling, with Tukey's test subsequently performed to compare each parameter. The rating scale data for ease of handling were averaged weekly, and assessed using the non-parametric Mann-Whitney $U$ test. The Steel-Dwass test, as a nonparametric test, was used to make comparisons between mice administrated diazepam and saline for each handling group for the two behavioral tests. The coefficient of variation $(\mathrm{CV})$ was calculated by dividing the standard deviation by the data mean multiplying the dividend by 100 . In all statistical tests, differences were considered significant at a probability level of $0.05(5 \%)$.

\section{RESULTS}

No sex differences were found for any of the parameters in this study; thus, data are shown for males and females combined. 


\section{Experiment 1}

Interaction tests. Overall, the voluntary interaction with the experimenter of mice handled by a tunnel was significantly greater than that of mice handled by the tail (ANOVA, $\mathrm{F}(1,78)=220.9, P<0.0001, \mathrm{n}=40$ per group) (Fig. 1). Mice picked up by the tail showed low voluntary interaction with the experimenter throughout this experiment.

At 4 weeks of age, mice tended to have more interaction by tunnel handling than by tail handling even on session 1 . Use of the tunnel remarkably increased the voluntary interaction with the experimenter. Significant differences between tunnel and tail handling continued to be detected from the second assessment on session 1 until the final session $(P<0.01)$. Voluntary interaction with the experimenter tended to be greater after than before handling, and significant differences were observed in the tunnel handling group in sessions 4 and $7(P<0.05)$. At 5 weeks of age, voluntary interaction with the experimenter tended to decrease in both handling groups from the start of daily oral administration to the final session. The decrease reached a significant difference in the tunnel handling group. In tunnel handling group, the significant differences were detected between the interaction before oral administration at session 8 and before oral administration at session $14(P<0.01)$, before oral administration at session 8 and after oral administration at session 11 and $14(P<0.01)$. However, voluntary interaction with tunnel handling (tunnel handling group) was greater than with the experimenter's hand (tail handling group) before and after administration in sessions 8,11 and $14(P<0.01)$. In addition, in the tunnel handling group, voluntary interaction with the experimenter before and after administration in sessions 14 was significantly greater than that before handling in session $1(P<0.01)$.

\section{Rating scale of ease of handling and excretion at handling}

Capturing and holding scores and excretion at handling are compared between tunnel and tail handling group ( $\mathrm{n}=40$ per group).

With respect to capturing scores, values tended to decrease at 5 weeks of age compared to 4 weeks of age (Table 1). The tunnel handling group was more easily captured than the tail handling group at 4 weeks $(P<0.01)$ and 5 weeks $(P<0.01)$ of age.

In holding scores, the tunnel handling group showed significantly less resistance than the tail handling group at 4 weeks $(P<0.01)$ and 5 weeks $(P<0.01)$ of age.

Through capturing and holding in 4 weeks of age, tunnel handling group showed significantly less number of urination $(P<0.05)$ and defecation $(P<0.01)$ compared to tail handling group $(\%$ handling events). Through capturing and oral administration in 5 weeks of age, tunnel handling group showed significantly less number of defecation $(P<0.05)$, but not urination.

\section{Experiment 2}

Behavioral tests. Table 2 shows results of the two behavioral tests for each handling group after intraperitoneal administration of saline or diazepam ( $\mathrm{n}=20$ per group).

In mice administrated saline, tunnel handling significantly increased the number of line crossings $(P<0.05)$ and time in inner area $(P<0.05)$ in the open field test. Furthermore, in mice administrated saline, the number of total arm entries $(P<0.05)$ and open

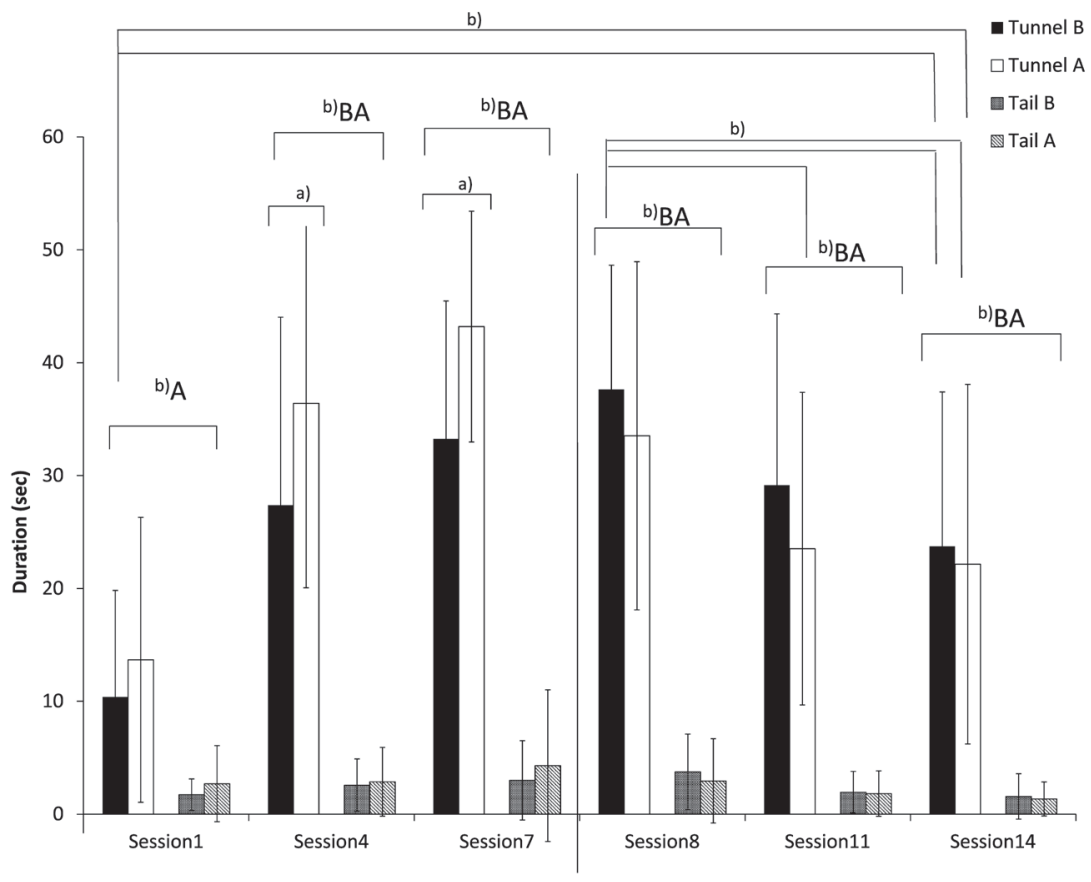

Fig. 1. Interaction to handling device of ICR mice subjected to tunnel and tail handling. B; before handling session, A; after handling session. Animals were acclimated in session 1 to session 7. Saline were administered orally to animals in session 8 to session 14. $\mathrm{n}=40$ animals per group. Values showed the means \pm SD. a); $P<0.05, \mathrm{~b}$ ); $P<0.01$. 
Table 1. Score of ease of handling and mouse urination and defecation during handling by different methods

(1) Capture-from the home cage

\begin{tabular}{lcr}
\hline \multicolumn{1}{c}{ Score of capturing } & Tunnel & \multicolumn{1}{c}{ Tail } \\
\hline Capturing in week 4 of age & $0.032 \pm 0.23^{\mathrm{b})}$ & $0.16 \pm 0.48^{\mathrm{b})}$ \\
Capturing in week 5 of age & $0.011 \pm 0.13^{\mathrm{b})}$ & $0.089 \pm 0.30^{\mathrm{b}}$ \\
\hline
\end{tabular}

(2) Holding-when being picked up and placed at hand or held for administration

\begin{tabular}{lrr}
\hline \multicolumn{1}{c}{ Score of holding } & Tunnel & \multicolumn{1}{c}{ Tail } \\
\hline Holding in week 4 of age & $0.075 \pm 0.46^{\mathrm{b})}$ & $0.91 \pm 1.33^{\mathrm{b})}$ \\
Administration in week of 5 age & $0.64 \pm 0.83^{\mathrm{b})}$ & $1.1 \pm 1.17^{\mathrm{b}}$ \\
\hline
\end{tabular}

(3) Urination and defecation

\begin{tabular}{lcc}
\hline \multicolumn{1}{c}{$\%$ handling events } & Tunnel & Tail \\
\hline Urination in week 4 of age & $0.36 \pm 0.22^{\mathrm{a})}$ & $2.5 \pm 0.69^{\mathrm{a})}$ \\
Urination in week 5 of age & $22.32 \pm 1.6$ & $21.43 \pm 1.3$ \\
Defecation in week 4 of age & $1.61 \pm 0.57^{\mathrm{b})}$ & $12.5 \pm 2.0^{\mathrm{b})}$ \\
Defecation in week 5 of age & $0.89 \pm 0.24^{\mathrm{a})}$ & $5.4 \pm 0.48^{\mathrm{a})}$ \\
\hline
\end{tabular}

Values showed the means \pm SD. $\mathrm{n}=40$ animals per group. a) $P<0.05$, b) $P<0.01$ tunnel vs tail.

Table 2. Behavior of mice and CV of parameter in open field and elevated plus maze tests

\begin{tabular}{|c|c|c|c|c|}
\hline \multirow{2}{*}{ Behavior } & \multicolumn{2}{|c|}{ SAL } & \multicolumn{2}{|c|}{$\mathrm{DZ}$} \\
\hline & Tunnel & Tail & Tunnel & Tail \\
\hline \multicolumn{5}{|l|}{ OFT } \\
\hline \multirow[t]{2}{*}{ Line crossing (n) } & $496.5 \pm 107.7^{\mathrm{a})}$ & $400.45 \pm 96.3^{\mathrm{a})}$ & $471.25 \pm 150.3$ & $376.8 \pm 230.0$ \\
\hline & 21.69 & 24.04 & 31.90 & 61.05 \\
\hline \multirow[t]{2}{*}{ Time in inner area (\%) } & $20.57 \pm 8.0^{\mathrm{a})}$ & $14.35 \pm 6.8^{\mathrm{a})}$ & $15.07 \pm 7.8$ & $13.1 \pm 9.2$ \\
\hline & 39.03 & 47.42 & 52.02 & 70.00 \\
\hline \multicolumn{5}{|l|}{ EPM } \\
\hline \multirow[t]{2}{*}{ Total entry (n) } & $28.1 \pm 4.6^{\mathrm{a})}$ & $21.8 \pm 7.9^{\mathrm{a})}$ & $30.2 \pm 13.9$ & $22.25 \pm 13.3$ \\
\hline & 16.30 & 36.25 & 45.95 & 59.79 \\
\hline \multirow[t]{2}{*}{ Open arm entry (n) } & $12.25 \pm 3.0^{\mathrm{a}, \mathrm{b})}$ & $10.7 \pm 5.0^{\mathrm{a})}$ & $19.9 \pm 8.5^{\mathrm{b})}$ & $15.65 \pm 8.6$ \\
\hline & 24.88 & 46.65 & 42.87 & 55.16 \\
\hline \multirow[t]{2}{*}{ Time on open arms $(\%)$} & $33.67 \pm 9.8^{\mathrm{a}, \mathrm{b})}$ & $39.15 \pm 13.0^{\mathrm{a}, \mathrm{b})}$ & $69.78 \pm 20.6^{b)}$ & $73.22 \pm 15.0^{\mathrm{b})}$ \\
\hline & 28.99 & 33.30 & 29.58 & 20.49 \\
\hline
\end{tabular}

Values showed the means \pm SD. $\mathrm{n}=20$ animals per group. a) $P<0.05$ tunnel vs tail, b) $P<0.05$ SAL vs DZ comparison tunnel handled. SAL, saline; DZ, diazepam; OFT, open field test; EPM, elevated plus maze.

arm entries $(P<0.05)$ were significantly greater in the tunnel handling group compared to the tail handling group in the elevated plus maze test. However, the \% time in open arms was significantly less in the tunnel handling group than the tail handling group $(P<0.05)$.

Mice administrated diazepam showed the same tendencies as those administrated saline. However, significant differences were not observed for all items. Diazepam increased the number of open arm entries $(P<0.05)$ and \% time in open arms $(P<0.05)$ in the both handling groups in the elevated plus maze test but diazepam did not show any significant difference in open field tests.

\section{Data variation}

Table 2 also shows calculation of CVs of the two behavioral tests for each handling group after intraperitoneal administration of saline or diazepam ( $n=20$ per group). According to the calculated parameters in the open field test and elevated plus maze test, CVs of all items, except $\%$ time in open arms of mice administrated diazepam, were greater in the tail handling group compared to the tunnel handling group. In addition, diazepam increased CVs of all items, except \% time in open arms.

\section{DISCUSSION}

As shown in previous studies, repeated tunnel handling increased voluntary interaction with the experimenter [6, 7]. However, repeated oral administration decreased voluntary interaction with the experimenter by mice. This decrease continued until the final session of oral administration; however, the interaction of mice to be handled by a tunnel was greater compared to mice subjected 
to tail handling. Therefore, mice preferred being tunnel handled rather than being handled by the tail in the present 1-week oral administration. Thus, motivation to approach human was increased by tunnel handling. In this study, many mice subjected to tunnel handling approached straight to a tunnel and gloved hand as soon as starts of interaction tests. Since animals had learned handling device (a tunnel and gloved hands) as somethings that capture and hold them, voluntary interaction to different objects between handling groups was similar as a measure of tameness in this respect.

It was expected that capture scores would increase following the negative memory of oral administration; however, the degree of capturing difficulty tended to be reduced at 5 weeks of age compared to 4 weeks of age in both handling groups. This might be attributable to the mice becoming acclimated to handling. Because of the escalation in procedural difficulty and resistance of mice, holding scores were greater at 5 weeks of age (handling plus oral administration) compared to those at 4 weeks of age (acclimation to handling). In regards to voluntary interaction with the experimenter, oral administration appears to have resulted in increased aversion of mice to the experimenter. Although oral administration elevated the aversion of mice, wildness that indicates the degree of handling difficulty was lower in the tunnel handling groups than in the tail handling groups for all procedures in this study. Thus, tunnel use could reduce workload to experimenter through facilitating handling of ICR mice. Goto et al. [5] suggested that less wildness is similar to "reluctance to avoid human". Judging from this suggestion and results of interaction tests, it is suggested that tameness was increased by tunnel handling. In addition, oral administration decreased voluntary interaction, which is similar to "motivation to approach to human", but did not decrease "reluctance to avoid human" when mice were captured. In a previous study, domesticated mice have been selected for "reluctance to avoid humans" and these strains were never selected for higher "motivation to approach humans" [5]. Our study corresponds to this study and indicated "motivation to approach human" is easy to increase by tunnel use at handling. Moreover, oral administration decrease "motivation to approach human" after the increase of tameness. As well as the results in a study of Hurst and West [7], tunnel handling decreased urination and defecation in this study. However, at oral administration by the same procedures in both groups, urination was remarkably increased and it covered effects of handling methods.

According to the elevated plus maze test in this study, mice subjected to tunnel handling preferred to stay in an enclosed area. The mice might have acquired such a preference through experiencing a tunnel. Thus, the experience of tunnels complicated measuring anxiety in the elevated plus maze test. In the open field test, the conflicting motives between a natural aversion to brightly lit areas and the drive to explore was exploited. A long duration of time in inner areas of the open field test indicates low anxiety [15]. In this study, tunnel handling decreased anxiety in open field test, but not elevated plus maze test. Exploratory activity was consistently greater in the tunnel handling group compared to the tail handling group in the elevated plus maze (the number of arm entries) and open field (the number of line crossings) tests. Diazepam significantly decreased anxiety in the elevated plus maze test, but not in the open field test. This is because the two main effects of diazepam that decrease anxiety and decrease activity are conflicted in the open field test. Prut and Belzung [14] reported that diazepam does not have consistent effects on anxiety as measured in the open field test, for example, effects of diazepam to anxiety-like behavior were increasing or decreasing or nothing. Therefore, the influence of diazepam on our two behavioral tests was normal.

According to calculated CVs, standard deviation normalized by the mean, indicated smaller variation in the tunnel handling group compared to the tail handling group in almost all items in the behavioral tests, regardless of what (saline or diazepam) was injected. Just as we expected, diazepam increased CVs of almost all parameters, but there was not the case with this (like \% time in open arms in this study), so more studies would be necessary including various items of behavioral tests. Our results suggest that tunnel handling might decrease data variation in pharmacological tests. ICR, it is outbred strain, can mimic the human population, which has heterozygous genetic background. It could more increase variation than inbred strain. It would be necessary to be repeated studies considering the number of animals, varieties of measurement item, various strains including inbred and outbred, in order to clear influence of tunnel use at handling on experimental variation.

In conclusion, mice handled using a tunnel showed a longer duration of voluntary interaction with the experimenter compared to mice picked up by the tail, in mice that received oral saline administration for 1 week following acclimation to handling for 1 week. Tunnel handling improved the evaluation of capture and hold during acclimation and oral administration. In addition, tunnel handling increased exploratory behavior in two behavioral tests and decreased anxiety in the open field test, but not in elevated plus maze test. Furthermore, mice handled by a tunnel showed the tendency of less data variation in the elevated plus maze and open field tests compared to mice picked up by the tail, in mice administrated saline or diazepam (similar to pharmacological studies). According to these results, a tunnel should be used to handle ICR mice in order to enhance animal welfare. Moreover, tunnel use facilitated handling of ICR mice.

\section{REFERENCES}

1. Ambrose, N. and Morton, D. B. 2000. The use of cage enrichment to reduce male mouse aggression. J. Appl. Anim. Welf. Sci. 3: 117-125. [CrossRef]

2. Balcombe, J. P., Barnard, N. D. and Sandusky, C. 2004. Laboratory routines cause animal stress. Contemp. Top. Lab. Anim. Sci. 43: 42-51. [Medline]

3. Bonnichsen, M., Dragsted, N. and Hansen, A. K. 2005. The welfare impact of gavaging laboratory rats. Animal Welfare -Potters Bar Then Wheathampstead 14: 223 .

4. Fridgeirsdottir, G. A., Hillered, L. and Clausen, F. 2014. Escalated handling of young C57BL/6 mice results in altered Morris water maze performance. Ups. J. Med. Sci. 119: 1-9. [Medline] [CrossRef]

5. Goto, T., Tanave, A., Moriwaki, K., Shiroishi, T. and Koide, T. 2013. Selection for reluctance to avoid humans during the domestication of mice. 
Genes Brain Behav. 12: 760-770. [Medline] [CrossRef]

6. Gouveia, K. and Hurst, J. L. 2013. Reducing mouse anxiety during handling: effect of experience with handling tunnels. PLoS One 8: e66401. [Medline] [CrossRef]

7. Hurst, J. L. and West, R. S. 2010. Taming anxiety in laboratory mice. Nat. Methods 7: 825-826. [Medline] [CrossRef]

8. Lockworth, C. R., Kim, S. J., Liu, J., Palla, S. L. and Craig, S. L. 2015. Effect of enrichment devices on aggression in manipulated nude mice. J. Am. Assoc. Lab. Anim. Sci. 54: 731-736. [Medline]

9. Maurer, B. M., Döring, D., Scheipl, F., Küchenhoff, H. and Erhard, M. H. 2008. Effects of a gentling programme on the behaviour of laboratory rats towards humans. Appl. Anim. Behav. Sci. 114: 554-571. [CrossRef]

10. Meijer, M. K., Sommer, R., Spruijt, B. M., van Zutphen, L. F. M. and Baumans, V. 2007. Influence of environmental enrichment and handling on the acute stress response in individually housed mice. Lab. Anim. 41: 161-173. [Medline] [CrossRef]

11. Moons, C. P., Van Wiele, P. and Ödberg, F. O. 2004. To enrich or not to enrich: providing shelter does not complicate handling of laboratory mice. Contemp. Top. Lab. Anim. Sci. 43: 18-21. [Medline]

12. Park, S. H., Ko, S. K. and Chung, S. H. 2005. Euonymus alatus prevents the hyperglycemia and hyperlipidemia induced by high-fat diet in ICR mice. J. Ethnopharmacol. 102: 326-335. [Medline] [CrossRef]

13. Price, E. O. 2002. Animal Domestication and Behavior. CABI Publishing, New York.

14. Prut, L. and Belzung, C. 2003. The open field as a paradigm to measure the effects of drugs on anxiety-like behaviors: a review. Eur. J. Pharmacol. 463: 3-33. [Medline] [CrossRef]

15. Schmitt, U. and Hiemke, C. 1998. Strain differences in open-field and elevated plus-maze behavior of rats without and with pretest handling. Pharmacol. Biochem. Behav. 59: 807-811. [Medline] [CrossRef]

16. Van Loo, P. L. P., Van Zutphen, L. F. M. and Baumans, V. 2003. Male management: Coping with aggression problems in male laboratory mice. Lab. Anim. 37: 300-313. [Medline] [CrossRef]

17. Wahlsten, D., Metten, P. and Crabbe, J. C. 2003. A rating scale for wildness and ease of handling laboratory mice: results for 21 inbred strains tested in two laboratories. Genes Brain Behav. 2: 71-79. [Medline] [CrossRef]

18. Zhong, S. Z., Ge, Q. H., Qu, R., Li, Q. and Ma, S. P. 2009. Paeonol attenuates neurotoxicity and ameliorates cognitive impairment induced by d-galactose in ICR mice. J. Neurol. Sci. 277: 58-64. [Medline] [CrossRef] 